\title{
Time variations of proton flux in the Earth inner radiation belt for 2006-2015 years based on the PAMELA and the ARINA data
}

\author{
V.V. Malakhov', S.Yu. Aleksandrin, S.V. Koldashov, A.G. Mayorov, M.A. Mayorova \\ on behalf of PAMELA collaboration. \\ National Research Nuclear University "MEPhI" \\ Moscow, Kashirskoe shosse, 31, Russian Federation \\ E-mail: vvmalakhov@mephi.ru
}

\begin{abstract}
The PAMELA and the ARINA experiments onboard satellite RESURS-DK1 are carried out since 2006 up to now. PAMELA instrument in the first place is intended to measure of high energy antiparticles in cosmic rays while main purpose of the ARINA instrument is study of high-energy charged particle bursts in the magnetosphere. Both these experiments have possibility to study protons in the inner radiation belt. Complex of these two instruments covers proton energy range from $30 \mathrm{MeV}$ up to trapping limit $(\mathrm{E}=\sim 2 \mathrm{GeV})$. Measurements with PAMELA and ARINA include both last minimum and maximum of solar cycle. It is important because existing empirical radiation belt models do not able to calculate trapped particle fluxes taking into account solar activity changing, e.g. widely used AP-8 model allows to evaluate proton fluxes just in two cases: for minimum or maximum of a solar activity. In this report we present temporal profiles of proton flux in the inner zone of the radiation belt $(1.11<\mathrm{L}<1.50,0.18<\mathrm{B}<0.22 \mathrm{G}$ for 2006 2015 year) based on PAMELA and ARINA measurements. Dependence of proton fluxes on degree of solar activity were studied for various phases of 23/24 solar cycle. At that it was shown that proton fluxes of energies $>30 \mathrm{MeV}$ at the solar minimum several times greater than at the solar maximum.
\end{abstract}

The 34th International Cosmic Ray Conference

30 July- 6 August, 2015

The Hague, The Netherlands

${ }^{1}$ Speaker 


\section{Introduction}

Knowledge about the behaviour of the inner radiation belt on its boundaries is important because it helps in better understanding a particle losses mechanisms. Though, in general, the inner radiation belt is a stable structure, its edges affected by various factors, such as changes in atmosphere density followed by solar activity changes [1], changes in the Earth magnetic lines position due to decreasing dipole moment. There were quite a lot of measurements in the Earth radiation belts starting from 60th, see for example [2][3], but none of them excluding POES programme [2] does not cover full solar cycle. In general there are unfilled holes in data on a low altitudes (low L-shells) of high energy particles E $>100 \mathrm{MeV}$, which affected by changes most.

\section{Instruments.}

\subsection{The RESURS-DK1 satellite}

The Resurs-DK1 satellite which carries the PAMELA and the ARINA instruments was launched on $15^{\text {th }}$ June 2006 into an elliptical orbit with altitudes of 350-610 km and an inclination of 70.4 degrees. The orbit was circularised on 10 Sep 2010 to constant $573 \mathrm{~km}$ altitude. At these altitudes the instrument crosses the radiation belt in the South Atlantic Anomaly zone 6-8 times a day, so it allows collecting enough data in this region to study trapped particle fluxes with high statistical accuracy.

\subsection{The PAMELA spectrometer}

The PAMELA instrument was designed for study of antiparticles in cosmic rays, but it also has possibilities to measure trapped particle flux of energies greater than $\sim 80 \mathrm{MeV}$. The PAMELA apparatus, as shown in figure 1, compounds of following subdetectors: a time of flight system (ToF), a magnetic spectrometer, an electromagnetic calorimeter, an anticoincidence system (CAS, CAT, CARD), a shower bottom scintillator and a neutron detector. In this analysis ToF and anticoincidence system was used. ToF produces a trigger signal if all of its planes are hit (i.e. it mainly registers protons with energy more than $80 \mathrm{MeV}$ ) and determines particle velocity and direction. For more detailed information see [4].

\subsection{The ARINA spectrometer}

The ARINA spectrometer originally was designed to study high-energy charged particle bursts in the magnetosphere. It consists of 10 scintillator plates and has capability to identify electrons and protons and to measure energy of particles by its range in a multilayer detector. Scheme of the ARINA instrument is shown in figure 2. Energy range of ARINA is $30-110 \mathrm{MeV}$. For more detailed information see in [5].

\section{Methods}

Different energy channels was performed by using different selection, $80<\mathrm{E}<100$ performed by selecting particles stopping in the last ToF plane, $100<\mathrm{E}<120$ by selecting particles stopping in first calorimeter plane, and $\mathrm{E}>300 \mathrm{MeV}$ for particles moving through whole instrument. For ARINA energy channel was determine by number of planes a particle went before stopping. Because fluxes in the Radiation belt are complicated and vary with different parameters such as L-shell, B (or B/B0), Pitch-angle, time (of f10.7 or sunspot number), several multidimensional nets was created out of measured values of fluxes in its nodes. There are several such experimental multidimensional shapes were constructed as f(E,L,B,Pitch,t), f(E,L,B/B0,Pitch,t), $\mathrm{f}(\mathrm{E}, \mathrm{L}, \mathrm{B} / \mathrm{B} 0$, Pitch,f10.7) etc. To get dependence showed below corresponding projections of this net was used. Results are in form of multidimension shape where along first 5 dimensions $\mathrm{E}, \mathrm{L}$, 
B/B0, Pitch anf f10.7 are used and sixth dimension are flux. To work with this multidimension histogram special routine was created.

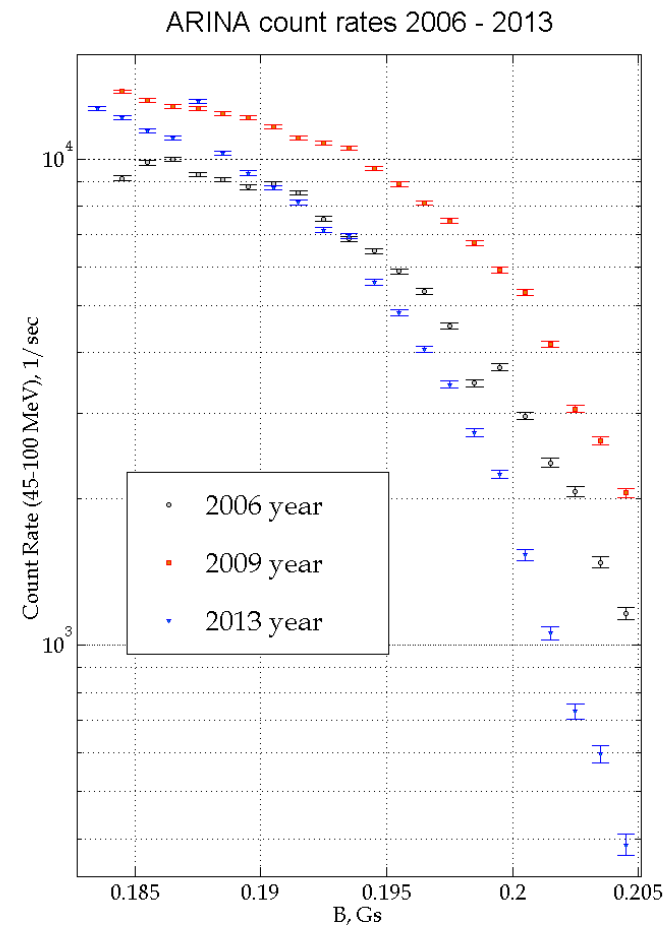

Figure 1. Proton flux vs B dependence measured by the ARINA for 2006 year (black), 2009 (red) and 2013 (blue) years
PAMELA count rates $2006-2014$

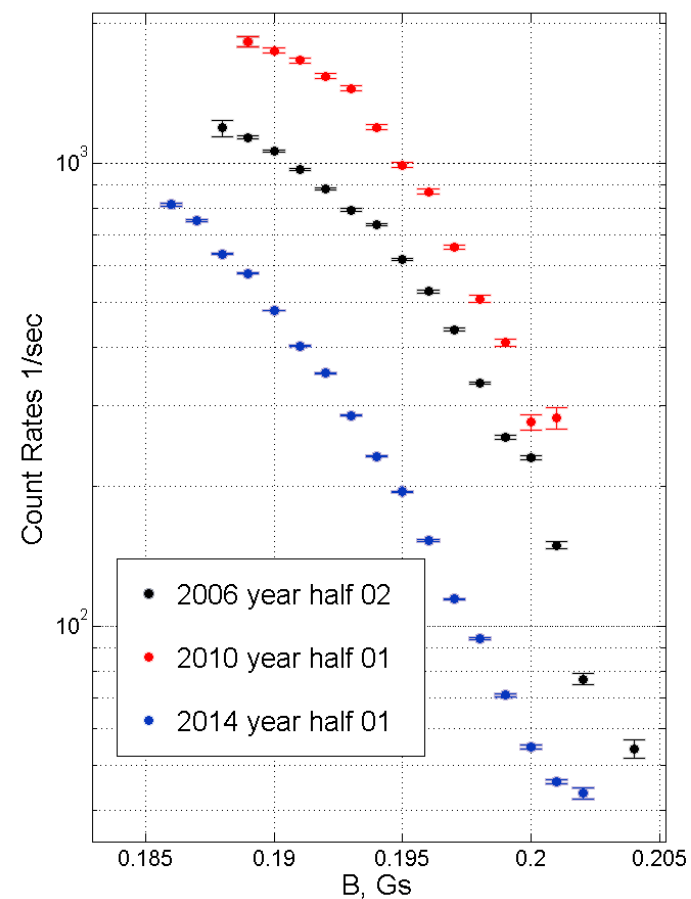

Figure 2. Proton flux vs B dependence measured by the PAMELA for 2006 (black), 2010 (red) and 2014 (blue) years.

\section{Results and discussion}

In the figure 1 and 2 shown proton flux dependence on B for different phases of solar cycle for the ARINA and the PAMELA correspondingly. It's seen that for maximum and minimum of solar cycle the ARINA and the PAMELA shown the same results 


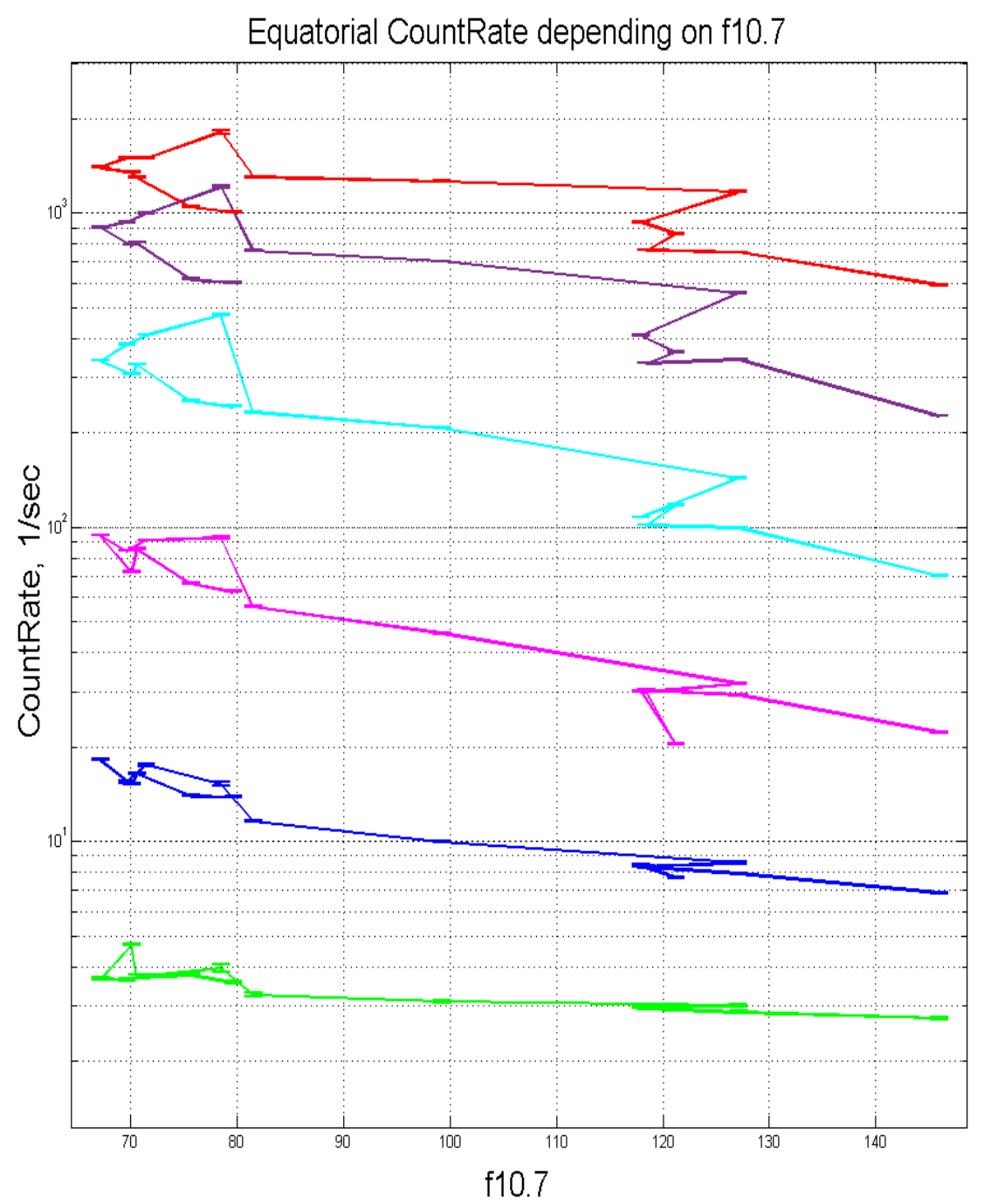

Fig. 3 proton flux dependence on $\mathrm{f} 10.7$

\section{Conclusion}

In this work time variations of proton fluxes with energies $\mathrm{E}>80 \mathrm{MeV}$ at the low edge of the inner radiation belt with PAMELA and ARINA instrument were obtained. An anti correlation between flux and level of the solar activity was shown. Flux dependence on B for different L-shells in interval $1.12-1.20$ has been obtained. Good agreement between PAMELA and ARINA data is seen.

\section{References}

[1] Miyoshi Y, Morioka A, Misawa H 2000 Long Term Modulation of Low Altitude Proton Radiation Belt by the Earth's Atmosphere Geophysical Research Letters 2714 2169-72 
[2] Huston S L, Kuck G A, Pfitzer K A 1997 Low Altitude Trapped Radiation Model Using TIROS/NOAA data Radiation Belts:Models and Standards

[3] Heynderickx D, Kruglanski M, Pierrard V, Lemaire J, Looper M D and Blake J B 1999 A low altitude trapped proton model for solar minimum conditions based on SAMPEX/PET data EEL Tram. Nucl. Sci. 46

[4] Picozza P, Galper A, Castellini G et al, 2007 PAMELA: Payload for Antimatter Matter Exploration and Light nuclei Astrophysics, Astropart. Phys. 27 296-315

[5] Bakaldin A et al, 2007, Satellite experiment ARINA for studying seismic effects in the highenergy particle fluxes in the Earth's magnetosphere, Cosmic Research, 45 445-8 